Supplemental information

\title{
Solvent-free Production of $\varepsilon$-Caprolactone from Oxidation of Cyclohexanone Catalyzed by Nitrogen Doped Carbon Nanotubes
}

Zhihui Cai ${ }^{1}$, Duo Liu ${ }^{1}$, Jiangnan Huang ${ }^{1}$, Jianning Feng ${ }^{1}$, Hongjuan Wang ${ }^{1}$, Guangxing Yang ${ }^{1}$, FengPeng ${ }^{2}$, Yonghai $\mathrm{Cao}^{1 *}, \mathrm{Hao} \mathrm{Yu}^{1 *}$

${ }^{1}$ School of Chemistry and Chemical Engineering, Guangdong Provincial Key Lab of Green Chemical Product Technology, South China University of Technology, Guangzhou, 510641, China

${ }^{2}$ Guangzhou Key Laboratory for New Energy and Green Catalysis, School of Chemistry and Chemical Engineering, Guangzhou University, Guangzhou 510006,

China

${ }^{3}$ School of Biology and Biological Engineering, South China University of Technology, Guangzhou, 510641, China

*Corresponding author:

E-mail: meyhcao@scut.edu.cn (Y. Cao);

yuhao@scut.edu.cn (H. Yu) 


\section{Table and Figure Captions}

Table S1 Catalytic performance of NCNTs on the Baeyer-Villiger oxidation with different RKA

Table S2 Comparison of the catalytic performance of various catalysts on the Baeyer-Villiger oxidation

Table S3 Influences of oxygen content of NCNTs on the Baeyer-Villiger oxidation

Table S4 Influence of oxygen content of NCNTs catalysts on Baeyer-Villiger oxidation

Figure S1 The stainless steel reactor with 2 L volume in this work.

Figure S2 (a) The recyclability of NCNT-3.38 on Baeyer-Villiger oxidation. Conditions: $1 \mathrm{MPa} \mathrm{O}_{2}, 35{ }^{\circ} \mathrm{C}, 1500 \mathrm{rpm}, 0.14 \mathrm{~mol} \mathrm{Cy}=\mathrm{O}, 0.035 \mathrm{~mol} \mathrm{BEA}, 30 \mathrm{mg}$ NCNTs, 1 h. Raman spectra (b) and TEM image (c) of recycled NCNTs-3.38.

Figure S3 Parameters influence on the reaction catalyzed by NCNTs-3.38 in a molar-scale reaction, (a) catalyst dosage, conditions: $1 \mathrm{MPa} \mathrm{O}_{2}, 40{ }^{\circ} \mathrm{C}, 1500 \mathrm{rpm}$, $5.43 \mathrm{~mol} \mathrm{Cy}=\mathrm{O}, 1.36$ mol BEA, 8 h. (b) reaction time, conditions: $1 \mathrm{MPa}_{2}, 40^{\circ} \mathrm{C}$, $1500 \mathrm{rpm}, 5.43 \mathrm{~mol} \mathrm{Cy}=\mathrm{O}, 1.36 \mathrm{~mol} \mathrm{BEA}, 195 \mathrm{mg}$ catalyst. (c) pressure, conditions: $40{ }^{\circ} \mathrm{C}, 1500 \mathrm{rpm}, 5.43 \mathrm{~mol} \mathrm{Cy}=\mathrm{O}, 1.36 \mathrm{~mol} \mathrm{BEA}, 100 \mathrm{mg}$ catalyst, $8 \mathrm{~h}$.

Figure S4 XPS survey of carbon catalysts with different N contents.

Figure S5 Raman spectra of carbon catalysts with different N contents.

Figure S6 Dependence of specific activity on different N species.

Figure S7 Dependence of specific activity on different specific N ratios.

Figure S8 XPS survey of NCNTs-3.38 refluxed with 9 mol/L $\mathrm{HNO}_{3}$ and anneal 
treatment.

Figure S9 Raman spectra of NCNTs-3.38 refluxed with 9 mol/L $\mathrm{HNO}_{3}$ and anneal treatment.

Figure S10 XPS survey of NCNTs-3.38 refluxed with 9 mol/L $\mathrm{HNO}_{3}$ and anneal treatment.

Figure S11 Raman spectra of NCNTs-3.38 refluxed with 9 mol/L $\mathrm{HNO}_{3}$ and anneal treatment.

Figure S12 High resolution N1s XPS spectra of NCNTs-3.38 underwent the HNO3-treatment and thermal annealing process in this work

Figure S13 (a) Dependence of specific activity on different $\mathrm{N}$ species. (b) Dependence of specific activity on different specific $\mathrm{N}$ ratios.

Figure S14 High resolution $\mathrm{N}_{1 \mathrm{~s}}$ XPS spectra of NCNTs underwent the HNO3-treatment and thermal annealing process in this work

Figure S15 (a) Dependence of specific activity on different $\mathrm{N}$ species. (b) Dependence of specific activity on different specific $\mathrm{N}$ ratios.

Figure S16 The relationship between the $\mathrm{HNO}_{3}$ treatment time and the $\mathrm{Np} / \mathrm{Ng}$ ratio. 
Table S1 Catalytic performance of NCNTs on the Baeyer-Villiger oxidation with

\begin{tabular}{|c|c|c|c|c|c|c|c|c|}
\hline \multirow{4}{*}{ Entry } & \multirow{4}{*}{$\begin{array}{l}\mathrm{Cy}=\mathrm{O} \\
(\mathrm{mmol})\end{array}$} & \multirow{4}{*}{$\begin{array}{c}\text { BEA } \\
(\mathrm{mmol})\end{array}$} & \multirow{4}{*}{$\begin{array}{c}\mathrm{R}_{\mathrm{s}}^{[\mathrm{b}]} \\
\left(\mathrm{mmol} / \mathrm{m}^{2} / \mathrm{h}\right)\end{array}$} & \multirow{4}{*}{$\begin{array}{c}\mathrm{R}_{\mathrm{m}}{ }^{[\mathrm{c}]} \\
(\mathrm{mmol} / \mathrm{g} / \mathrm{h})\end{array}$} & \multirow{4}{*}{$\begin{array}{l}\mathrm{Cy}=\mathrm{O} \\
\text { Conv. } \\
(\%)\end{array}$} & \multirow{4}{*}{$\begin{array}{c}\varepsilon-\mathrm{CL} \\
\text { Yield }(\%)\end{array}$} & \multirow{4}{*}{$\begin{array}{c}\varepsilon-\mathrm{CL} \\
(\mathrm{mmol})\end{array}$} & \multirow{4}{*}{$\mathrm{AE}^{[\mathrm{d}]}$} \\
\hline & & & & & & & & \\
\hline & & & & & & & & \\
\hline & & & & & & & & \\
\hline 1 & 117 & 39 & 31.58 & 4676.37 & 18.71 & 15.20 & 23.38 & 0.59 \\
\hline 2 & 130 & 65 & 36.56 & 5413.84 & 24.17 & 20.43 & 27.07 & 0.52 \\
\hline 3 & 88 & 88 & 43.53 & 6446.46 & 37.92 & 34.71 & 32.23 & 0.44 \\
\hline
\end{tabular}

${ }^{\text {a] }}$ Conditions: $1 \mathrm{MPa} \mathrm{O}_{2}, 35^{\circ} \mathrm{C}, 5 \mathrm{mg}$ catalyst, $1 \mathrm{~h}, 1500 \mathrm{rpm}$. NCNTs is represented as NCNTs $-3.38 .{ }^{[b]}$ Initial rate of $\mathrm{Cy}=\mathrm{O}$ consumption per $\mathrm{m}^{2}$ of catalyst surface area.

${ }^{[c]}$ Initial rate of $\mathrm{Cy}=\mathrm{O}$ consumption per gram of catalyst. ${ }^{[\mathrm{d}]}$ Aldehyde efficiency, calculated by (produced molar amount of $\varepsilon-\mathrm{CL}) /($ consumed molar amount of BEA). 
Table S2 Comparison of the catalytic performance of various catalysts on the Baeyer-Villiger oxidation

\begin{tabular}{|c|c|c|c|c|c|c|c|c|}
\hline Entry & Catalyst & $\begin{array}{l}\mathrm{S}_{\mathrm{BET}} \\
\left(\mathrm{m}^{2} / \mathrm{g}\right)\end{array}$ & $\begin{array}{c}\mathrm{R}_{\mathrm{s}}{ }^{\mathrm{a}]} \\
\left(\mathrm{mmol} / \mathrm{m}^{2} / \mathrm{h}\right)\end{array}$ & $\begin{array}{c}\mathrm{R}_{\mathrm{m}}^{[\mathrm{b}]} \\
(\mathrm{mmol} / \mathrm{g} / \mathrm{h})\end{array}$ & $\begin{array}{c}\mathrm{Cy}=\mathrm{O} \\
\text { Conv. } \\
(\%)\end{array}$ & $\begin{array}{c}\varepsilon-C L \\
\text { Yield (\%) }\end{array}$ & $\begin{array}{c}\varepsilon-\mathrm{CL} \\
(\mathrm{mmol})\end{array}$ & $\mathrm{AE}^{[\mathrm{c}]}$ \\
\hline 1 & $\mathrm{c}-\mathrm{MWCNTs}^{[\mathrm{d}]}$ & 76.8 & 0.32 & 24.75 & 99.00 & 99.00 & 1.98 & - \\
\hline 2 & Graphite $^{[\mathrm{e}]}$ & 15.9 & 7.27 & 115.63 & 92.50 & 92.50 & 9.25 & - \\
\hline 3 & Ketjen black ${ }^{[\mathrm{f}]}$ & 1270.0 & 0.10 & 122.00 & 61.00 & 61.00 & 2.44 & 0.73 \\
\hline 4 & NHPI $[\mathrm{g}]$ & - & - & - & 100.00 & 99.60 & 2.00 & 0.48 \\
\hline 5 & $\mathrm{~m}-\mathrm{Zr} \mathrm{P}^{[\mathrm{h}]}$ & 400.0 & 0.13 & 53.06 & 78.00 & 78.00 & 7.80 & - \\
\hline 6 & Co-HMS-X ${ }^{[i]}$ & 674.0 & 0.01 & 9.36 & 93.60 & 92.70 & 1.87 & - \\
\hline
\end{tabular}

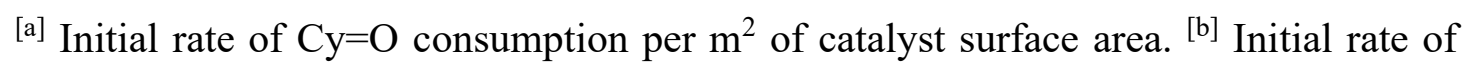
$\mathrm{Cy}=\mathrm{O}$ consumption per gram of catalyst. ${ }^{[c]}$ Aldehyde efficiency, calculated by (produced molar amount of $\varepsilon-\mathrm{CL}) /\left(\right.$ consumed molar amount of BEA). ${ }^{[\mathrm{d}]}$ Conditions: $10 \mathrm{mg}$ c-MWCNTs, $2 \mathrm{mmol} \mathrm{Cy}=\mathrm{O}, 4 \mathrm{mmol}$ BEA, $10 \mathrm{~mL}$ DCE, $\mathrm{O}_{2}$ balloon, $50{ }^{\circ} \mathrm{C}, 8 \mathrm{~h}$ (ref 1). ${ }^{[\mathrm{e}]}$ Conditions: $10 \mathrm{mmol} \mathrm{Cy}=\mathrm{O}, 20 \mathrm{mmol} \mathrm{BEA}, 20 \mathrm{ml}$ 1,2-dichloroethane, 10 $\mathrm{mL} / \mathrm{min} \mathrm{O}_{2}, 20 \mathrm{mg}$ catalyst, $25{ }^{\circ} \mathrm{C}, 4 \mathrm{~h}$. (ref 2). ${ }^{[\mathrm{f}]}$ Conditions: $4.0 \mathrm{mmol} \mathrm{Cy}=\mathrm{O}, 4.0$ mmol BEA, $5 \mathrm{mg}$ carbon catalyst, $10 \mathrm{~mL}$ 1,2-dichloroethane, $\mathrm{O}_{2}, 50{ }^{\circ} \mathrm{C}, 4 \mathrm{~h}$ (ref 3). ${ }^{[\mathrm{g}]}$ Conditions: 2 mmol Cy=O, 4 mmol BEA, 10 mol \% NHPI, 20 mL 1,2-dichloroethane, $4.5 \mathrm{~h}, \mathrm{O}_{2}$ balloon, $40{ }^{\circ} \mathrm{C}$ (ref 4). ${ }^{[\mathrm{h}]}$ Conditions: $10 \mathrm{mmol} \mathrm{Cy}=\mathrm{O}, 17.5$ mmol BEA, 98 $\mathrm{mg} \mathrm{m}-\mathrm{ZrP}$, room temperature, $1.5 \mathrm{~h}, 10 \mathrm{~mL} / \mathrm{min} \mathrm{O}_{2}$ (ref 5). ${ }^{[i]}$ Conditions: $2 \mathrm{mmol}$ $\mathrm{Cy}=\mathrm{O}, 6 \mathrm{mmol} \mathrm{BEA}, 10 \mathrm{~mL} \mathrm{1,2-dichloroethane,100} \mathrm{mg} \mathrm{catalyst,} 10 \mathrm{~mL} / \mathrm{min} \mathrm{O}_{2}, 2 \mathrm{~h}$, $50{ }^{\circ} \mathrm{C}($ ref 6$)$ 
Table S3 Influences of oxygen content of NCNTs on the Baeyer-Villiger oxidation ${ }^{\text {[a] }}$

\begin{tabular}{|c|c|c|c|c|c|c|c|c|c|c|c|}
\hline $\begin{array}{c}\mathrm{HNO}_{3} \\
\text { refluxing time } \\
{[\mathrm{b}](\mathrm{h})} \\
\end{array}$ & $\begin{array}{c}\text { Annealing } \\
\text { Temperature } \\
\left({ }^{\circ} \mathrm{C}\right)\end{array}$ & $\begin{array}{c}\mathrm{O}^{[\mathrm{c}]} \\
(\text { at.\%) }\end{array}$ & $\begin{array}{l}\mathrm{N}^{[\mathrm{c}]} \\
(\text { at.\%) }\end{array}$ & $\begin{array}{l}\mathrm{S}_{\mathrm{BET}} \\
\left(\mathrm{m}^{2}\right. \\
\left.\mathrm{g}^{-1}\right)\end{array}$ & $\mathrm{I}_{\mathrm{D}} / \mathrm{I}_{\mathrm{G}}{ }^{[\mathrm{d}]}$ & $\begin{array}{c}\mathrm{R}_{\mathrm{s}}^{[\mathrm{e}]} \\
\left(\mathrm{mmol} / \mathrm{m}^{2} / \mathrm{h}\right)\end{array}$ & $\begin{array}{c}\mathrm{R}_{\mathrm{m}}^{[\mathrm{f}]} \\
(\mathrm{mmol} / \mathrm{g} / \mathrm{h})\end{array}$ & $\begin{array}{c}\mathrm{Cy}=\mathrm{O} \\
\text { Conv. }(\%)\end{array}$ & $\begin{array}{c}\varepsilon-C L \\
\text { Yield }(\%)\end{array}$ & $\begin{array}{c}\varepsilon-\mathrm{CL} \\
(\mathrm{mmol})\end{array}$ & $\mathrm{AE}^{[\mathrm{g}]}$ \\
\hline 1 & none & 2.39 & 3.26 & 168.96 & 0.65 & 5.70 & 962.88 & 3.54 & 2.81 & 3.8 & 0.76 \\
\hline 5 & none & 4.66 & 3.52 & 154.76 & 0.84 & 6.92 & 1071.68 & 3.94 & 3.12 & 4.2 & 0.82 \\
\hline 7 & none & 4.75 & 3.42 & 156.33 & 0.73 & 6.91 & 1079.84 & 3.97 & 3.22 & 4.4 & 0.83 \\
\hline 7 & 600 & 2.85 & 2.21 & 156.29 & 0.69 & 31.80 & 4969.44 & 18.27 & 13.87 & 18.9 & 0.71 \\
\hline
\end{tabular}

${ }^{[a]}$ Condition: $1 \mathrm{MPa} \mathrm{O}_{2}, 35{ }^{\circ} \mathrm{C}, 1500 \mathrm{rpm}, 136 \mathrm{mmol} \mathrm{Cy}=\mathrm{O}, 36$ mmol BEA, $5 \mathrm{mg}$ catalyst, $1 \mathrm{~h}$, NCNTs is represented as NCNTs-3.38. ${ }^{[\mathrm{b}]}$ Reflux conditions: 9 mol/L $\mathrm{HNO}_{3}, 85^{\circ} \mathrm{C} .{ }^{[c]}$ XPS survey can be found in Figure S8. ${ }^{[\mathrm{d}]}$ Raman spectral results could be found in Figure S9. ${ }^{[\mathrm{e}]}$ Initial rate of $\mathrm{Cy}=\mathrm{O}$ consumption per $\mathrm{m}^{2}$ of catalyst surface area. ${ }^{[\mathrm{f}]}$ Initial rate of $\mathrm{Cy}=\mathrm{O}$ consumption per gram of catalyst. ${ }^{[\mathrm{g}]}$ Aldehyde efficiency, calculated using (produced molar amount of $\varepsilon-\mathrm{CL}) /($ consumed molar amount of BEA). 
Table S4 Influence of oxygen content of NCNTs catalysts on Baeyer-Villiger oxidation ${ }^{\text {[a] }}$

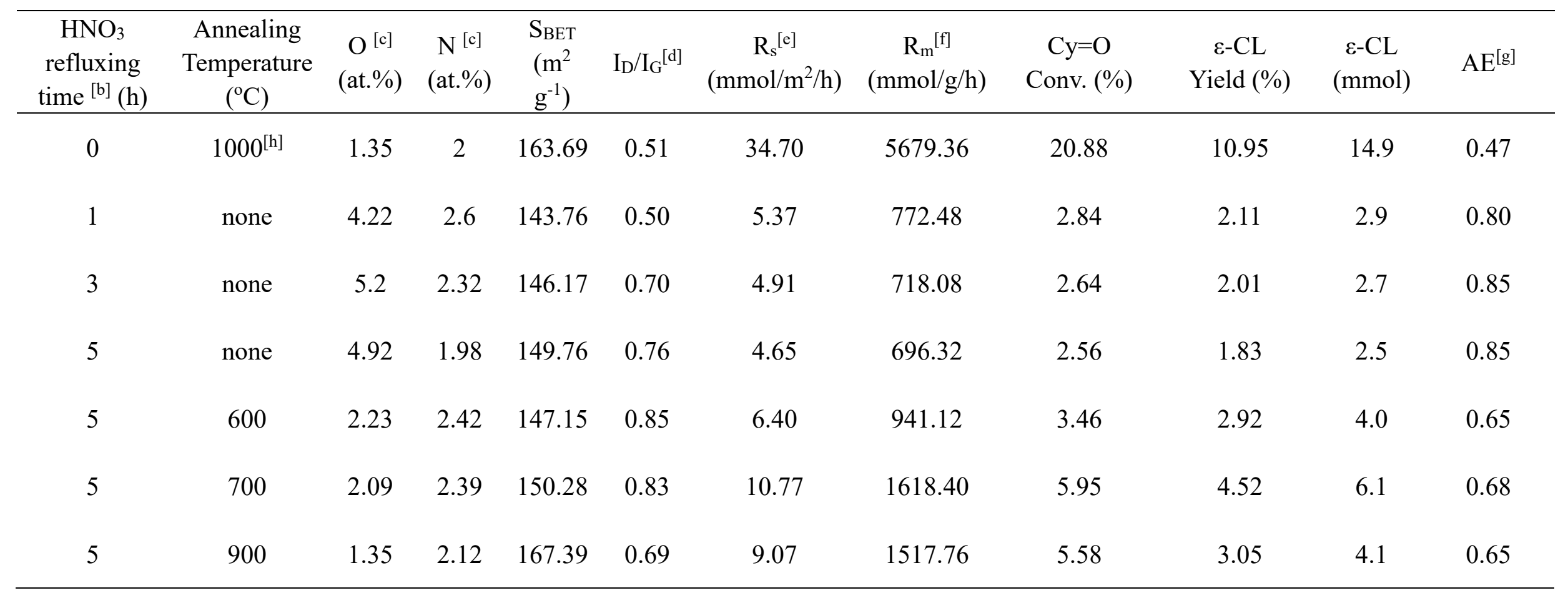

${ }^{[a]}$ Condition: $1 \mathrm{MPa} \mathrm{O}_{2}, 35{ }^{\circ} \mathrm{C}, 1500 \mathrm{rpm}, 136 \mathrm{mmol} \mathrm{Cy}=\mathrm{O}, 36$ mmol BEA, $5 \mathrm{mg}$ catalyst, $1 \mathrm{~h}$, NCNTs is represented as NCNTs-3.38. ${ }^{[\mathrm{b}]}$ Reflux conditions: 9 mol/L $\mathrm{HNO}_{3}, 85{ }^{\circ} \mathrm{C} .{ }^{[\mathrm{c}]}$ XPS survey can be found in Figure S10. ${ }^{[\mathrm{d}]}$ Raman spectral results was found in Figure S11. ${ }^{[\mathrm{e}]}$ Initial rate of $\mathrm{Cy}=\mathrm{O}$ consumption per $\mathrm{m}^{2}$ of catalyst surface area. ${ }^{[\mathrm{f}]}$ Initial rate of $\mathrm{Cy}=\mathrm{O}$ consumption per gram of catalyst. ${ }^{[\mathrm{g}]}$ Aldehyde efficiency, calculated using (produced molar amount of $\varepsilon$-CL)/(consumed molar amount of BEA). ${ }^{[\mathrm{h}]}$ Annealed under $1000{ }^{\circ} \mathrm{C}$ and $\mathrm{Ar}$ atmosphere for $2 \mathrm{~h}$. 


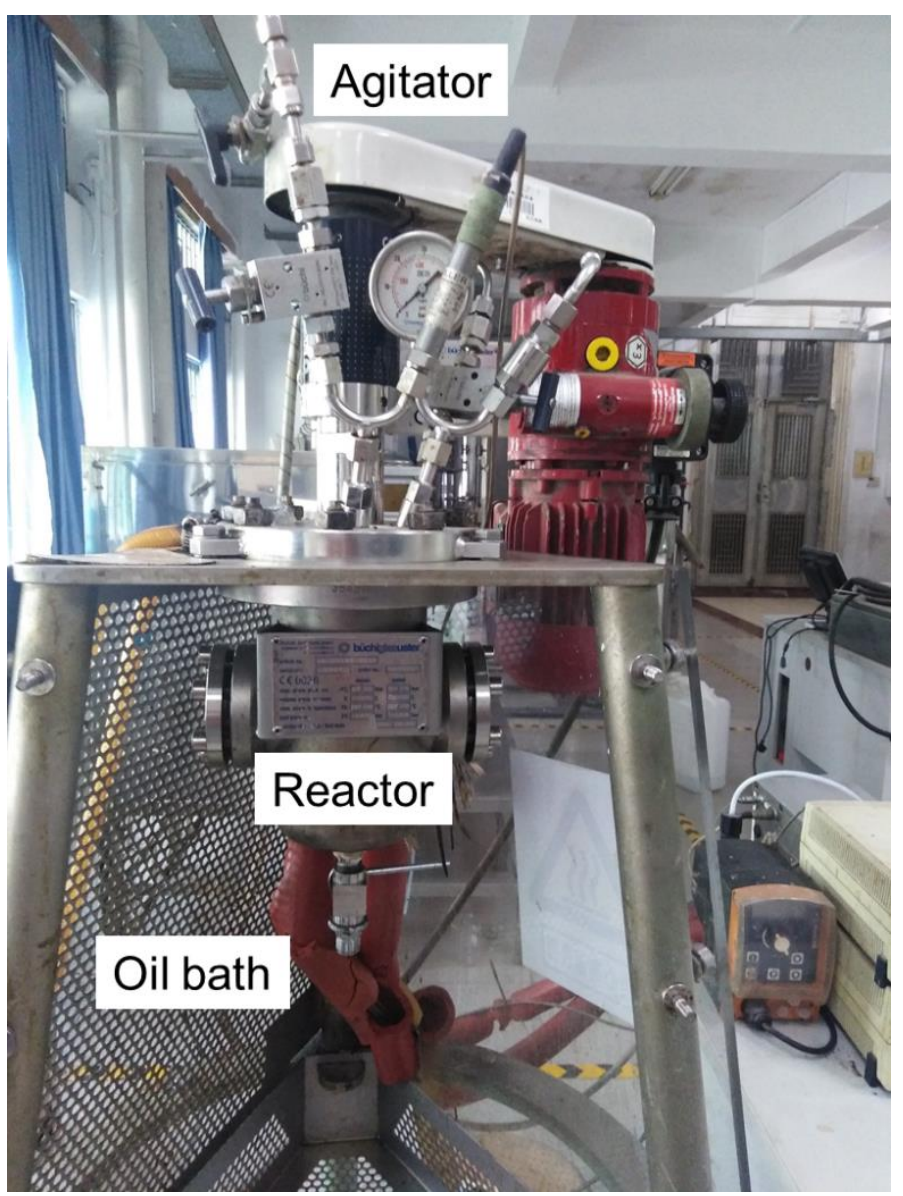

Figure S1 The stainless steel reactor with 2 L volume in this work. 
(a)

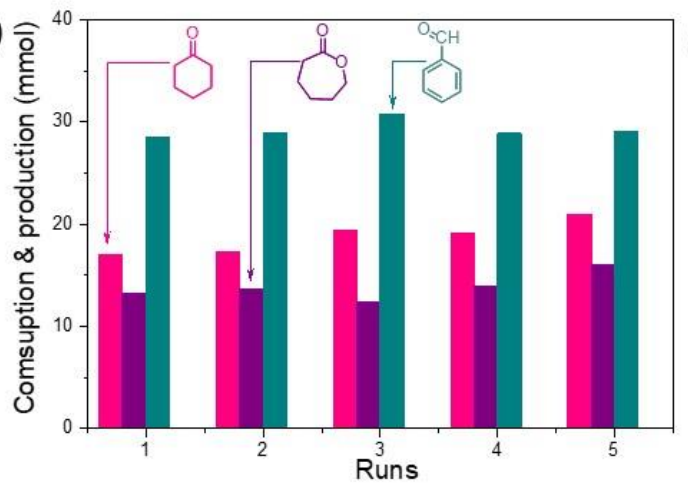

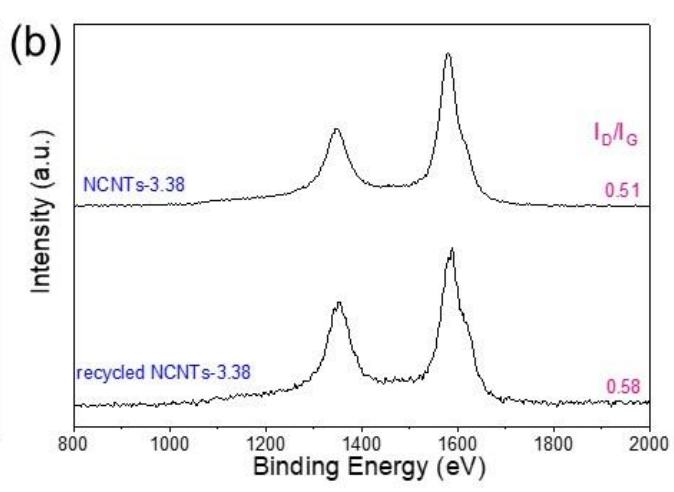

(c)

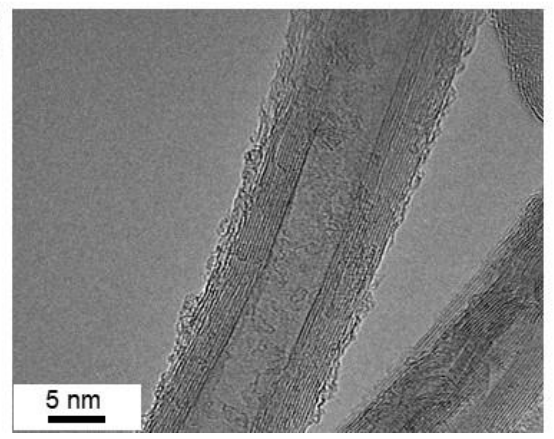

Figure S2 (a) The recyclability of NCNT-3.38 on Baeyer-Villiger oxidation.

Conditions: $1 \mathrm{MPa} \mathrm{O}_{2}, 35^{\circ} \mathrm{C}, 1500 \mathrm{rpm}, 0.14 \mathrm{~mol} \mathrm{Cy}=\mathrm{O}, 0.035 \mathrm{~mol} \mathrm{BEA}, 30 \mathrm{mg}$ catalyst, $1 \mathrm{~h}$. Raman spectra (b) and TEM image (c) of recycled NCNTs-3.38. 

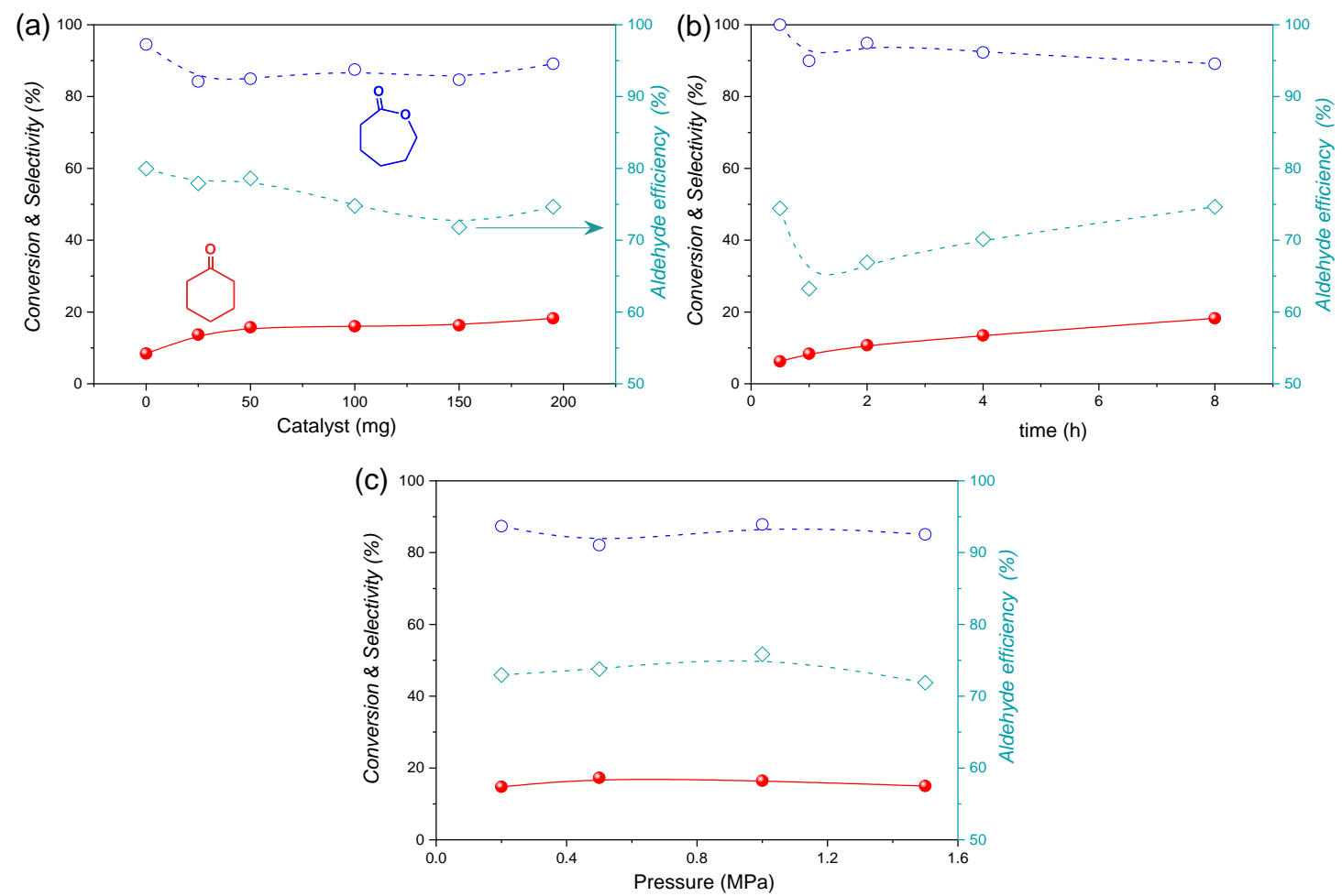

Figure S3 Parametric influence on the reaction catalyzed by NCNTs-3.38 in a molar-scale reaction. (a) catalyst dosage, conditions: $1 \mathrm{MPa}_{2}, 40{ }^{\circ} \mathrm{C}, 1500 \mathrm{rpm}$, $5.43 \mathrm{~mol} \mathrm{Cy}=\mathrm{O}, 1.36$ mol BEA, $8 \mathrm{~h}$. (b) reaction time, conditions: $1 \mathrm{MPa} \mathrm{O}_{2}, 40^{\circ} \mathrm{C}$, $1500 \mathrm{rpm}, 5.43 \mathrm{~mol} \mathrm{Cy}=\mathrm{O}, 1.36 \mathrm{~mol} \mathrm{BEA}, 195 \mathrm{mg}$ catalyst. (c) pressure, conditions: $40{ }^{\circ} \mathrm{C}, 1500 \mathrm{rpm}, 5.43 \mathrm{~mol} \mathrm{Cy}=\mathrm{O}, 1.36 \mathrm{~mol}$ BEA, $100 \mathrm{mg}$ catalyst, $8 \mathrm{~h}$. 


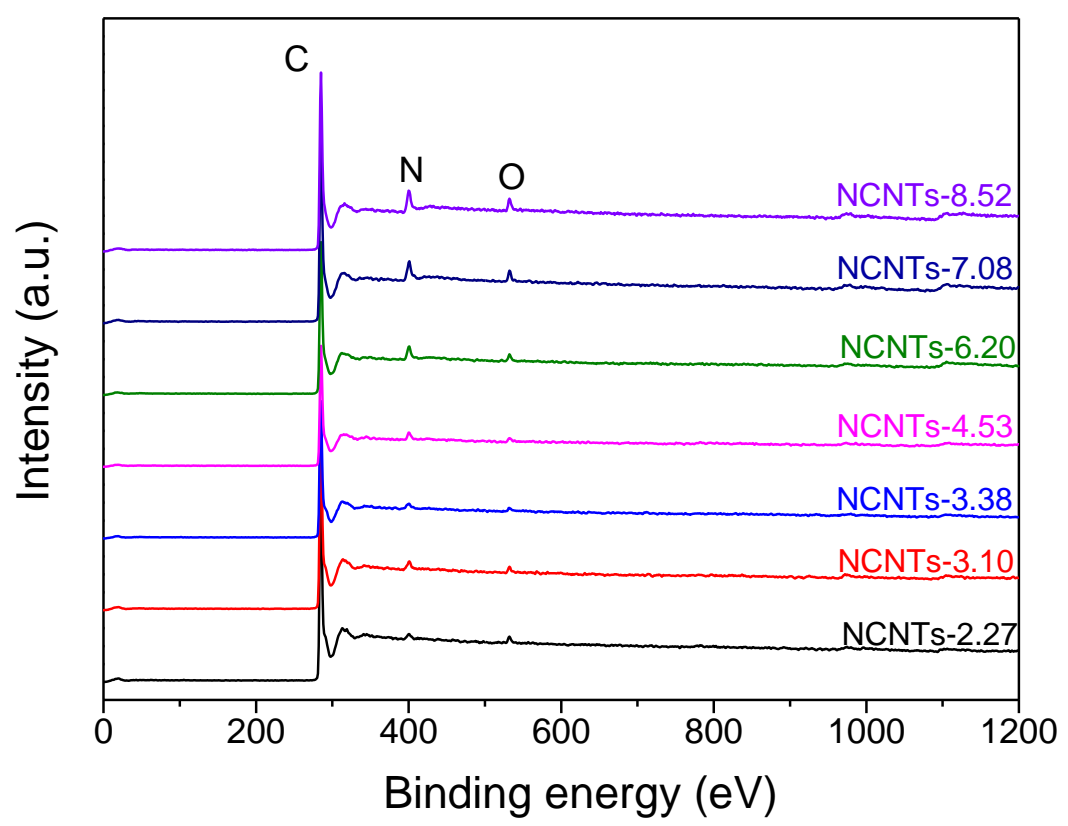

Figure S4 XPS survey of carbon catalysts with different N contents. 


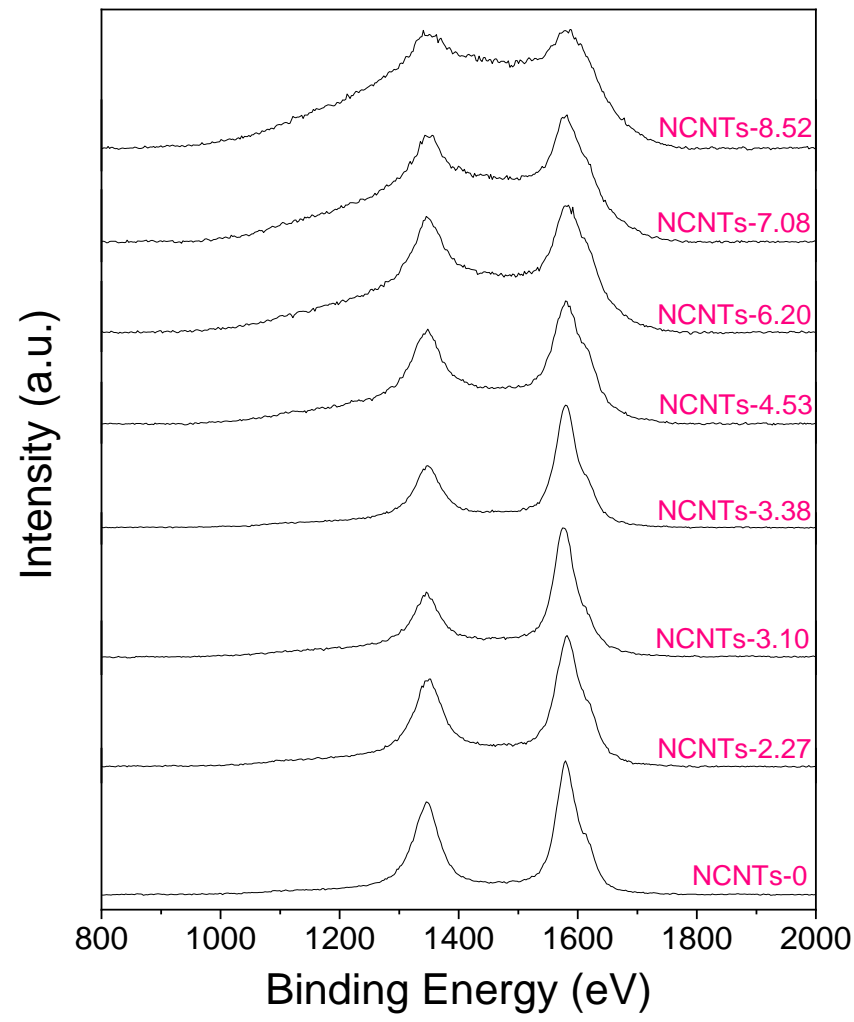

Figure S5 Raman spectra of carbon catalysts with different N contents. 


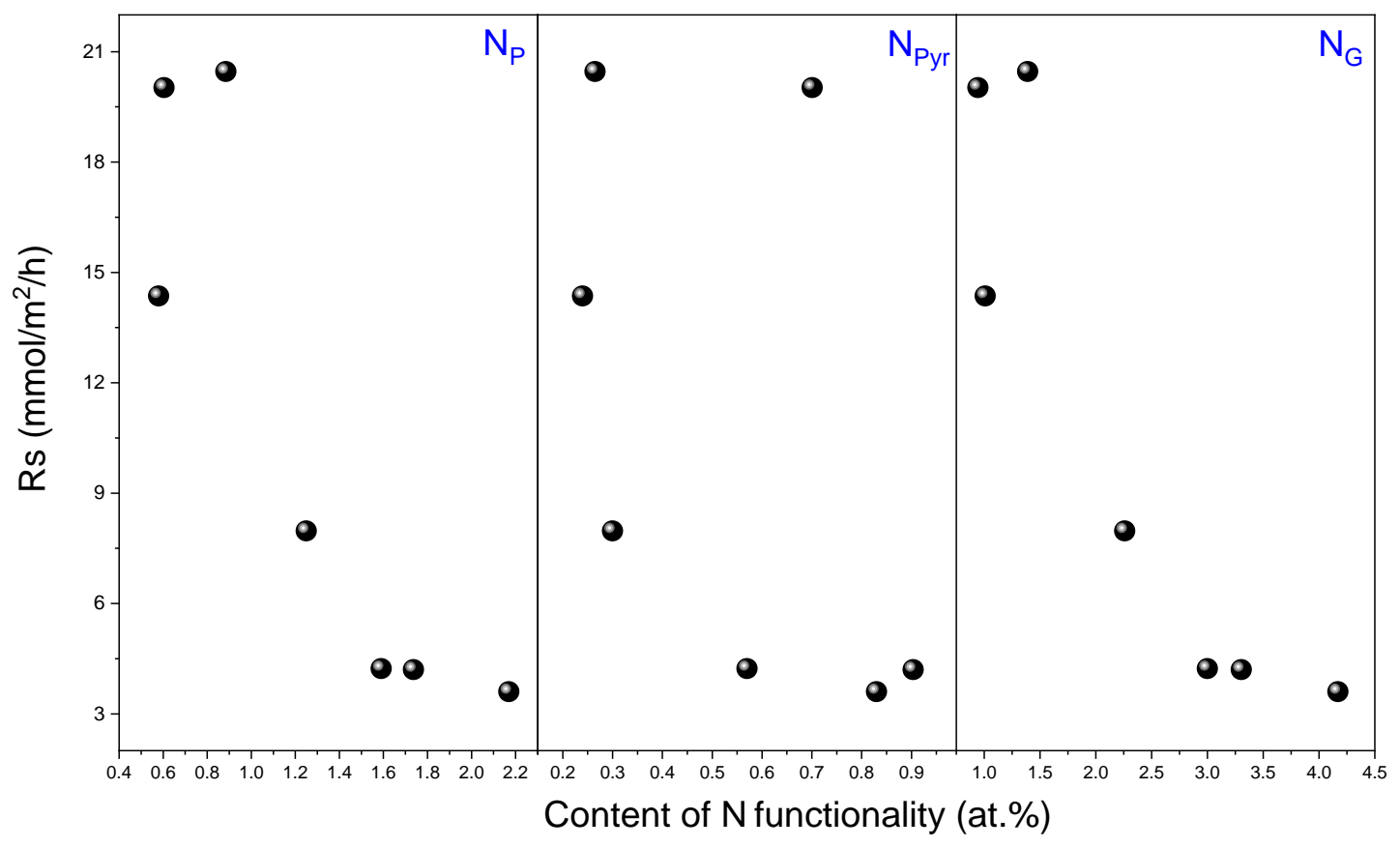

Figure S6 Dependence of specific activity on different $\mathrm{N}$ species. 


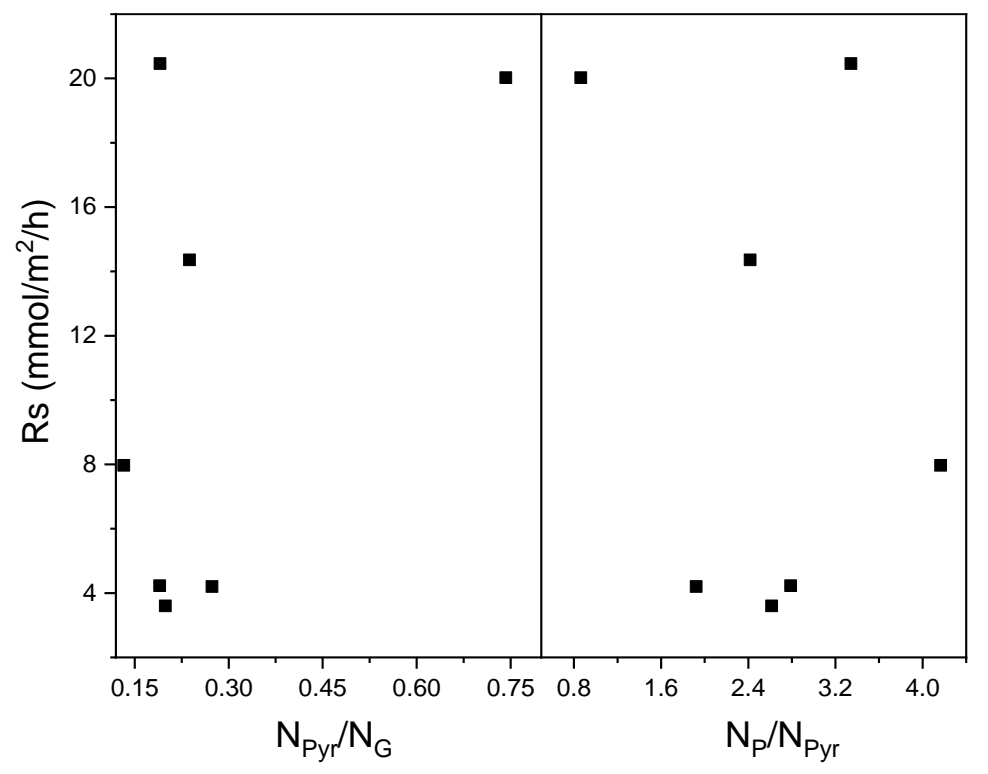

Figure S7 Dependence of specific activity on different specific $\mathrm{N}$ ratios. 


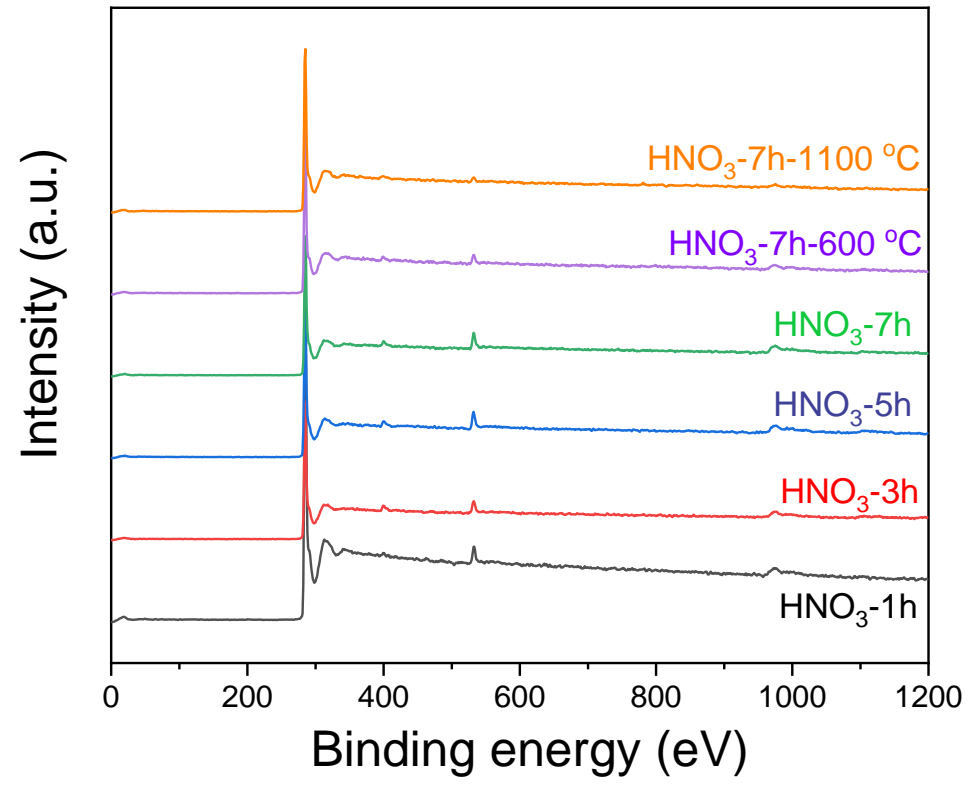

Figure S8 XPS survey of NCNTs-3.38 refluxed with 9 mol/L $\mathrm{HNO}_{3}$ and anneal treatment. 


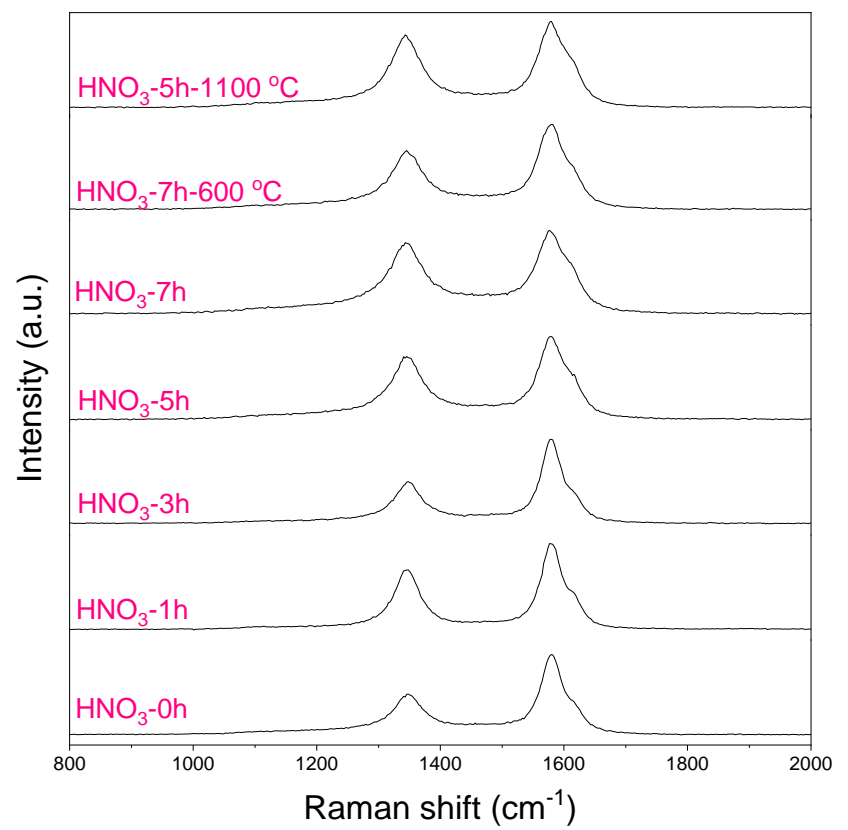

Figure S9 Raman spectra of NCNTs-3.38 refluxed with 9 mol/L $\mathrm{HNO}_{3}$ and anneal treatment. 


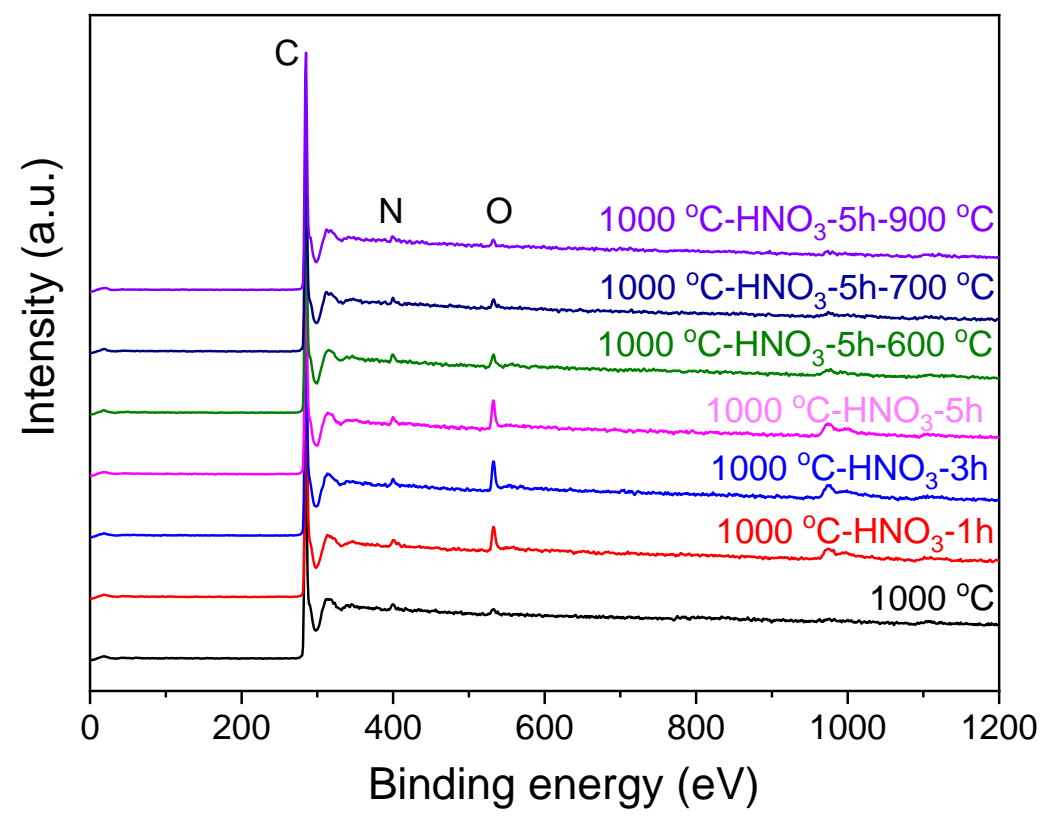

Figure S10 XPS survey of NCNTs-3.38 refluxed with 9 mol/L $\mathrm{HNO}_{3}$ and anneal treatment. 


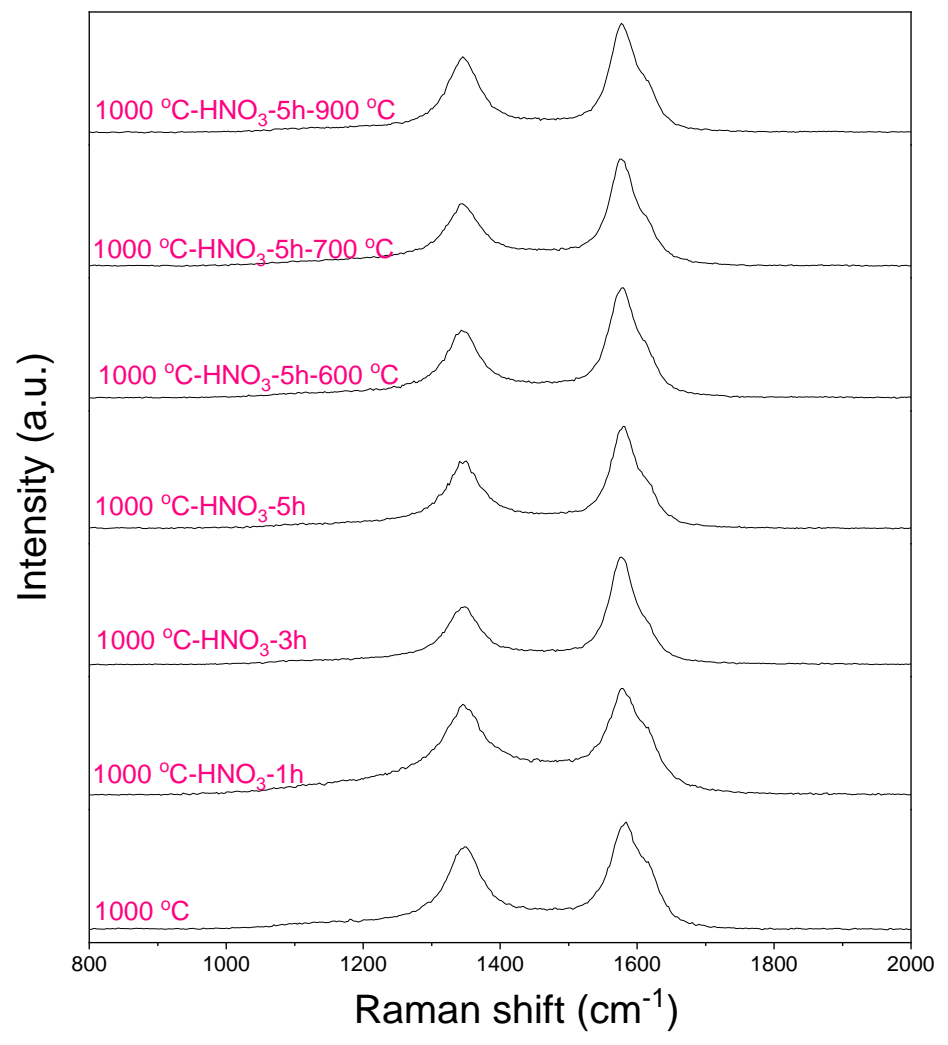

Figure S11 Raman spectra of NCNTs-3.38 refluxed with 9 mol/L $\mathrm{HNO}_{3}$ and anneal treatment. 


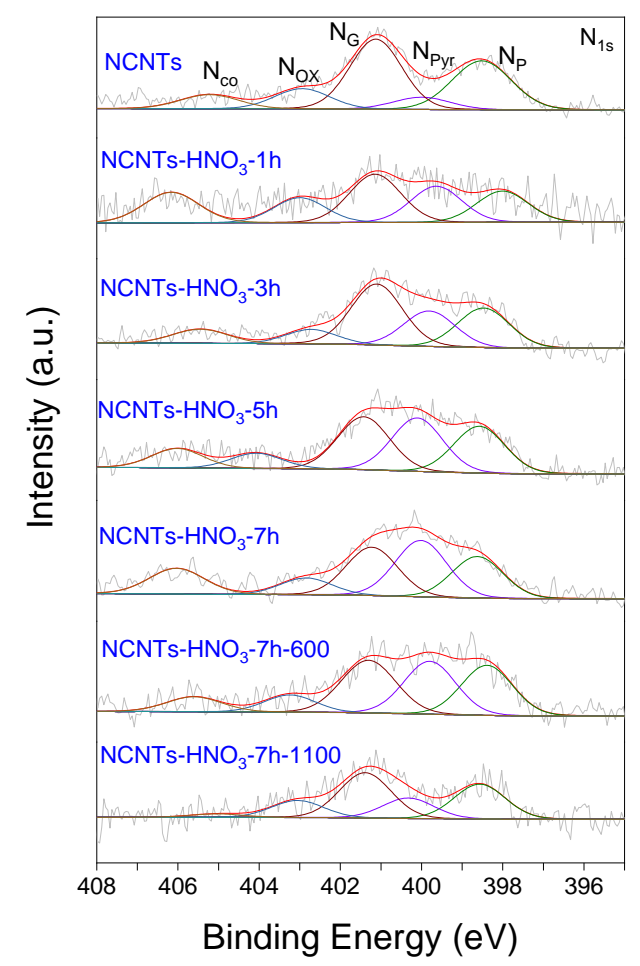

Figure S12 High resolution $\mathrm{N}_{1 \mathrm{~s}}$ XPS spectra of NCNTs-3.38 underwent the $\mathrm{HNO}_{3}$-treatment and thermal annealing process in this work 

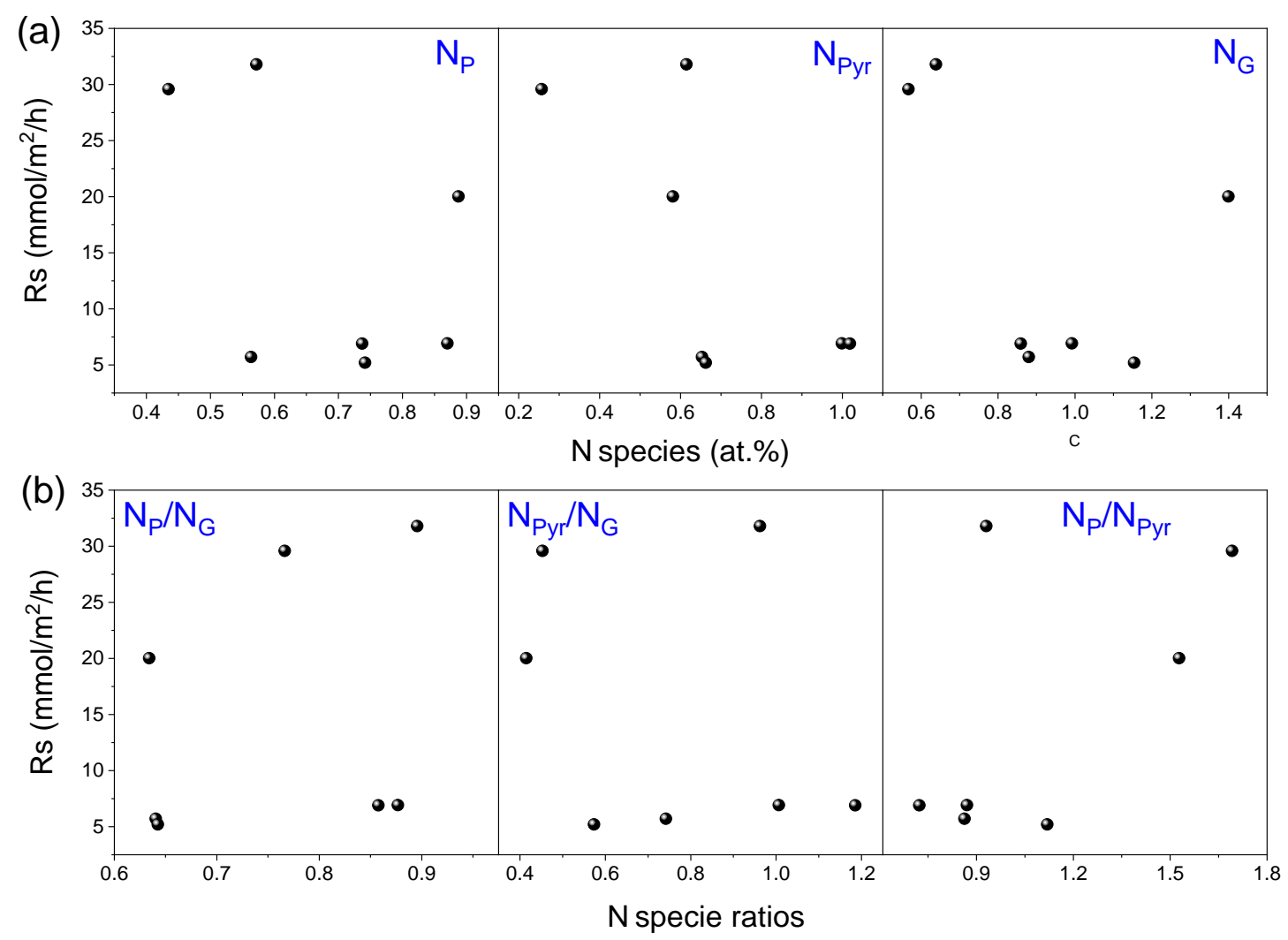

Figure S13 (a) Dependence of specific activity on different N species. (b) Dependence of specific activity on different specific $\mathrm{N}$ ratios. 


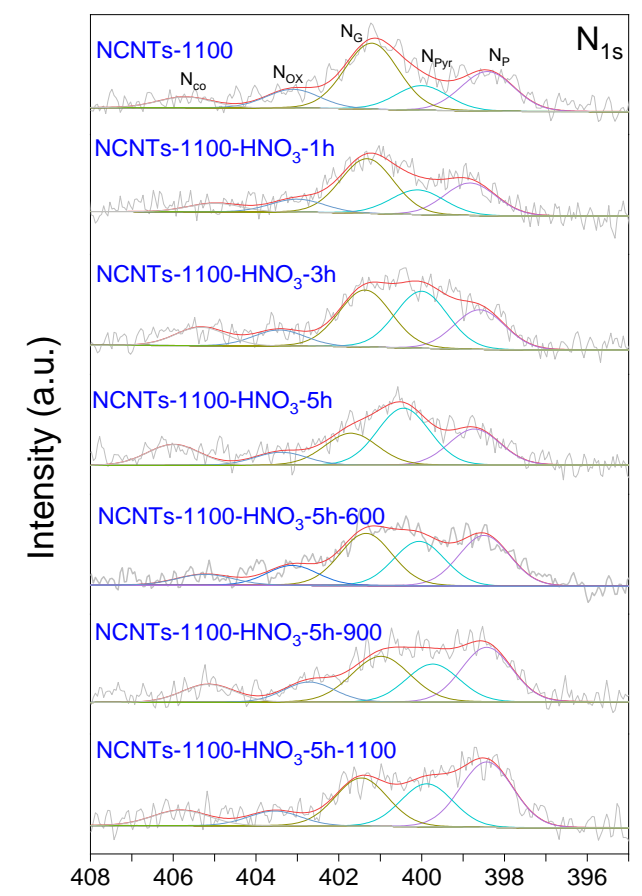

Figure S14 High resolution $\mathrm{N}_{1 \mathrm{~s}}$ XPS spectra of NCNTs underwent the $\mathrm{HNO}_{3}$-treatment and thermal annealing process in this work 

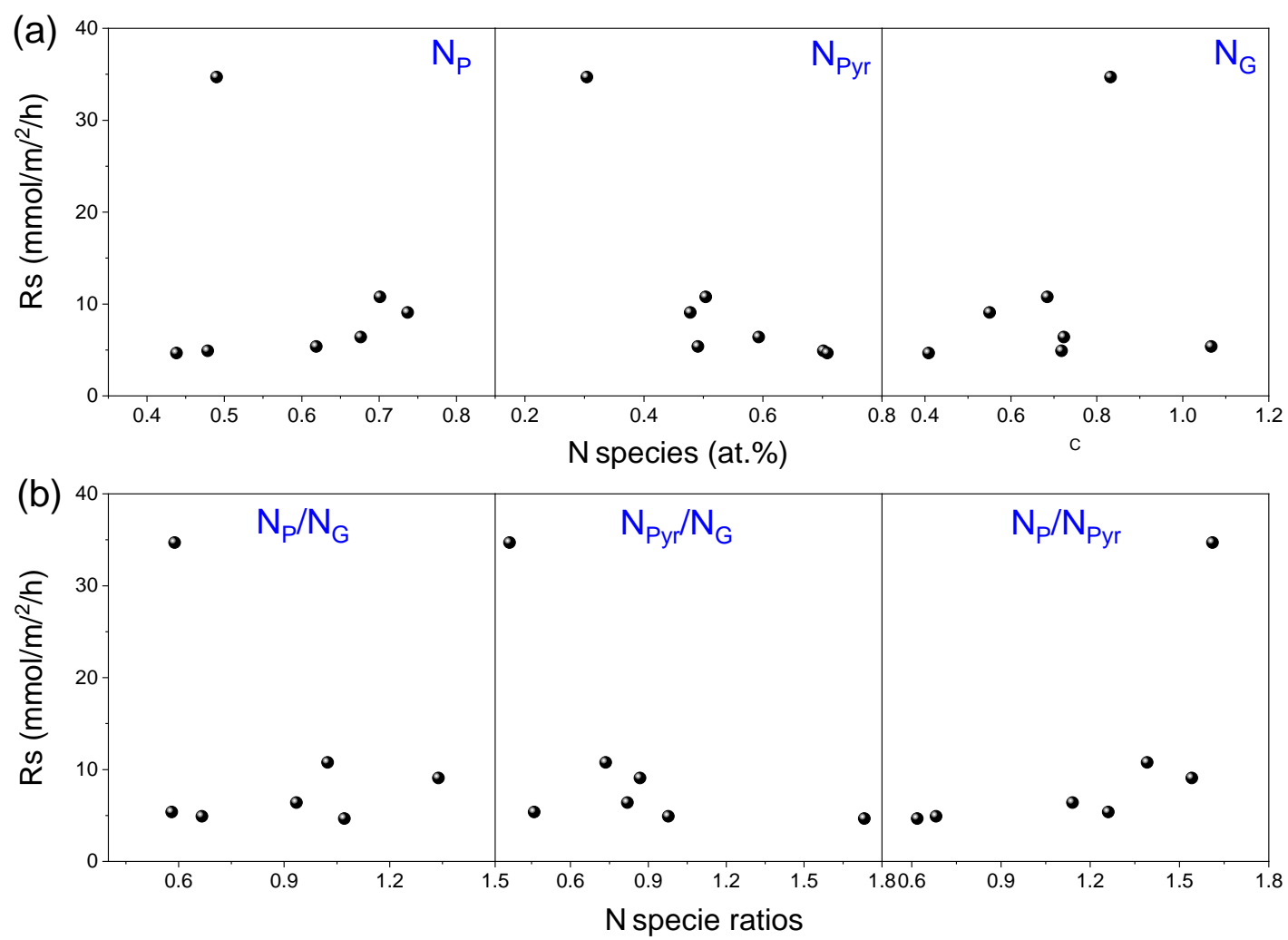

Figure S15 (a) Dependence of specific activity on different N species. (b)

Dependence of specific activity on different specific $\mathrm{N}$ ratios. 


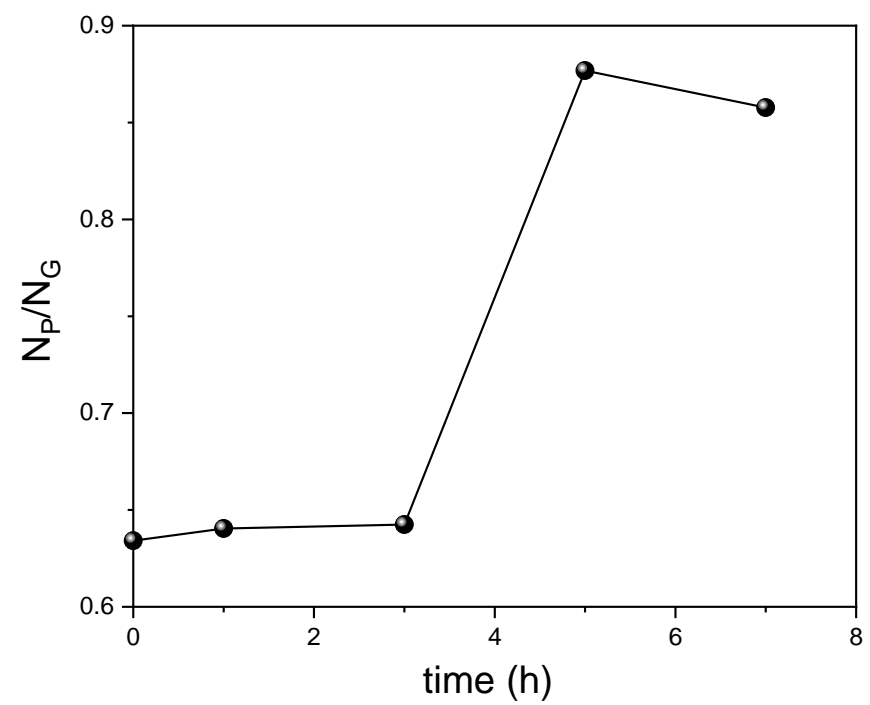

Figure S16 The relationship between the $\mathrm{HNO}_{3}$ treatment time and the $\mathrm{Np} / \mathrm{Ng}$ ratio. 


\section{References}

1. Chen, S.-Y.; Zhou, X.-T.; Wang, J.-X.; Luo, R.-C.; Luo, Q.-J.; Yu, L.-J.; Ji, H.-B. Promoting the aerobic Baeyer-Villiger oxidation of ketones over carboxylic multi-walled carbon nanotubes. Mol. Catal. 2017, 438, 152-158.

2. Li, Y.; Guo, M.; Yin, S.; Chen, L.; Zhou, Y.; Qiu, R.; Au, C. Graphite as a highly efficient and stable catalyst for the production of lactones. Carbon 2013, 55 (0), 269-275.

3. Nabae, Y.; Rokubuichi, H.; Mikuni, M.; Kuang, Y.; Hayakawa, T.; Kakimoto, M.-a. Catalysis by Carbon Materials for the Aerobic Baeyer-Villiger Oxidation in the Presence of Aldehydes. ACS Catal. 2013, 3 (2), 230-236.

4. Wang, L.; Wang, Y.; Du, R.; Dao, R.; Yuan, H.; Liang, C.; Yao, J.; Li, H. N-Hydroxyphthalimide (NHPI) Promoted Aerobic Baeyer-Villiger Oxidation in the Presence of Aldehydes. ChemCatChem 2018, 10 (21), 4961-4966.

5. Sinhamahapatra, A.; Sinha, A.; Pahari, S. K.; Sutradhar, N.; Bajaj, H. C.; Panda, A. B. Room temperature Baeyer-Villiger oxidation using molecular oxygen over mesoporous zirconium phosphate. Catal. Sci. Tech. 2012, 2 (11), 2375-2382.

6. Rahman, S.; Enjamuri, N.; Gomes, R.; Bhaumik, A.; Sen, D.; Pandey, J. K.; Mazumdar, S.; Chowdhury, B. Aerobic Baeyer-Villiger oxidation of cyclic ketones over periodic mesoporous silica $\mathrm{Cu} / \mathrm{Fe} / \mathrm{Ni} / \mathrm{Co}-\mathrm{HMS}-\mathrm{X}$. Appl. Catal. A: Gen 2015, 505, 515-523. 